\title{
DIGITALIS AND INTRACTABLE HEART FAILURE
}

\author{
BY \\ EVAN FLETCHER AND C. F. BRENNAN \\ From the Belfast City Hospital \\ Received June 5, 1957
}

Judicious digitalis therapy still remains the most important factor in the management of congestive heart failure at all its stages. Digitalis acts directly on the heart muscle, increasing the output of the failing heart in the presence of congestion, thereby restoring cardiac compensation (Harvey et al., 1949). As a result, therapeutic doses slow the pulse. Dosage required for adequate digitalization cannot be predicted with certainty, although body weight may be a guide (Eggleston, 1915). Therapeutic trial alone may indicate if digitalis is likely to be beneficial. Moreover, experience shows that digitalis requirements may vary from time to time during the course of congestive heart failure: any procedure that helps to determine these for patients requiring urgent help is a significant therapeutic advance, as it provides a basis for rational therapy. We have used the digitalis tolerance test (Lown and Levine, 1955) in assessing the digitalis requirements of nine cases of intractable congestive heart failure. Therapy based on the results benefited the patients, and allowed us to make certain deductions, which we believe to be important, about the nature of disturbances of rhythm occurring in congestive failure and their management.

It may be opportune at this stage to examine some of the important factors that play a role in the evolution of intractable congestive failure in so far as they affect digitalis therapy. A profound relationship exists between potassium metabolism and digitalis toxicity (Lown et al., 1951) with regard to the action of the drug on isolated groups of cardiac cells producing ectopic foci. Depletion of myocardial potassium sensitizes the heart to digitalis, so narrowing the margin between its therapeutic and toxic action. Potassium loss may render a therapeutic dose a toxic dose. During the development of congestive failure, total body potassium is reduced (Burrows and Sisson, 1950). A negative potassium balance is also favoured by nutritional problems in advanced congestive failurepoor appetite, monotonous diets, nausea, impaired absorption, and deranged liver function. Perhaps most important of all, therapeutic measures aimed at reducing the work of the heart may be responsible for substantial potassium loss, e.g. rigid salt restriction and administration of ammonium chloride during mercurial diuresis (Lesser et al., 1952). In addition diamox (Berliner et al., 1951), steroid hormones (Lown et al., 1951), ion exchange resins, and mechanical fluid drainage (paracentesis, acupuncture) deplete body stores of potassium. It would appear that the above factors play an important part in the evolution of intractable congestive failure as they may adversely modify the response of the heart to digitalis. In this connection also special reference must be made to coronary arterial disease. There is experimental evidence to show that myocardial ischæmia is associated with loss of intracellular myocardial potassium (Dennis and Moore, 1938). The progressive loss of myocardial potassium associated with advancing coronary disease may help to explain the changing sensitivity of the heart to digitalis. Toxic doses of digitalis may also deplete the myocardial cells of potassium (Calhoun and Harrison, 1931) and potassium administration will abolish toxic arrhythmias caused by digitalis (Sampson et al., 1943). We have utilized the complex relationship between digitalis and potassium metabolism in the management of our cases.

This paper is concerned with the particular problem of the digitalis status of patients admitted to hospital with intractable heart failure. Enquiry often reveals a long history of congestive failure, often for months or years, with varying response to digitalis and diuretic treatment. Frequently the 
dosage of digitalis given before admission cannot be ascertained, or the drug may have been omitted some time before the patient is seen, on account of some complicating factor. We submit that the decision to administer more digitalis or to withhold the drug in such cases amounts to a medical emergency. Our results with the acetyl strophanthidin test support the view that many of these cases can be regarded as cases of "failed digitalis treatment." When adequate digitalization is instituted striking improvement may occur. Clinical assessment alone has limitations. The physician may be hard put to differentiate the nausea and vomiting of congestion of the abdominal viscera from the more ominous sickness of digitalis intoxication due to the central emetic action of the drug (Hatcher and Weiss, 1922). Tachycardia certainly does not exclude serious digitalis intoxication (Heyl, 1932; Levine, 1948) and it may be impossible to decide even with electrocardiographic analyses if ectopic beats are toxic in origin or due to underlying organic heart disease. A trial dose of the usual digitalis preparations may be hazardous in the presence of potential digitalis intoxication. At any rate the correct dosage is conjectural, oral therapy may be ineffective on account of nausea and vomiting, and resort to intravenous administration of digitalis in a patient who may have already received an unknown quantity of the drug is not justifiable. Even the most rapidly acting preparations, ouabain, digoxin, and lanatocide $C$ (cedilanid) may produce toxic effects in these cases (Lown and Levine, 1955). Nevertheless to withhold digitalis from a patient who urgently requires it may have as serious consequences as administration of the drug in the presence of digitalis toxicity. The acetyl strophanthidin test (Lown and Levine, 1955) seems to have answered the question of digitalis requirements in our cases of intractable congestive failure.

Acetyl strophanthidin is a synthetic ester of strophanthidin, an aglycone obtained from the seeds of strophanthus kombé. It is prepared ready for intravenous use as an alcoholic solution, each ml. containing $0.5 \mathrm{mg}$. of the drug (approximately equivalent to 3 cat units. Eli Lilly and Co.). Its pharmacological action is the same as that of the digitalis glycosides (Chen and Elderfield, 1942; Gold et al., 1946; Greiner and Reilly, 1952; Root and Chen, 1953). However, the onset of its action is extremely rapid, and the commencement of its effect is noted within 5 minutes, 85 per cent within 10 minutes, and the full effect of the drug is almost completely dissipated within 4 hours. Thus acetyl strophanthidin can produce very rapid digitalization over a short period of time, which can be observed electrocardiographically.

\section{Technique for Acetyl Strophanthidin Test}

A solution of acetyl strophanthidin in 5 per cent glucose and water was prepared for intravenous injection, $0.5 \mathrm{mg}$. in every $10 \mathrm{ml}$. Saline solutions were not used, to avoid sodium administration in congestive heart failure. A drop of heparin was added to the syringe to prevent clotting. $2 \mathrm{ml}$. of the solution were administered every 5 minutes through the needle in an arm vein. Electrocardiographic records of an appropriate lead were taken at suitable intervals using a direct writer electrocardiograph. Occasionally the doses of acetyl strophanthidin were increased or given at shorter time intervals as warranted by electrocardiographic observation. Administration of the drug was stopped immediately if a therapeutic effect or a toxic effect was observed on the electrocardiograph, or if nausea occurred. Slowing of the heart rate was taken to indicate a therapeutic effect, and the production of abnormal rhythms a toxic effect. Interpretation of the test depended upon the amount of acetyl strophanthidin required to produce a result, and the nature of change in heart rate and rhythm. Throughout the period of observation procaine amide was available in a syringe for intravenous administration in the event of digitalis toxicity of severe degree.

\section{CASE REPORTS}

Case 1. Digitalis indicated. A man, aged 77 years, was admitted with advanced congestive heart failure due to coronary artery disease. Congestive heart failure had been present for six months, and he had received digitalis in varying doses with mercurial diuretics. Because of nausea and vomiting he dreaded taking digitalis. On examination the pulse was rapid (rate 123) and irregular. There was gallop rhythm, and evidence of pulmonary œdema. B.P. 150/90. An electrocardiogram 
appeared to confirm the presence of complete left bundle-branch block, and sinus rhythm complicated by the occurrence of very frequent ventricular extrasystoles of varying shape, usually considered typical of digitalis intoxication (Scherf and Schott, 1953). Serum potassium 4.5 m.eq. per litre. On account of the intense nausea and vomiting, and the occurrence of multiform ventricular extrasystoles, and the patient's apparent "intolerance" to oral digitalis (he refused to take the drug), it was decided that no further digitalis should be given. During the succeeding five days his condition deteriorated, the pulse remained rapid, and attacks of cardiac asthma became more frequent. The patient was persuaded to co-operate. $0.25 \mathrm{mg}$. was administered orally every six hours. After twenty-four hours tachycardia with pulsus bigeminus occurred, and severe nausea and vomiting. The digitalis was promptly stopped and potassium chloride $5 \mathrm{~g}$. administered daily by mouth in divided doses to combat what appeared to be digitalis intoxication. Two days later the patient's condition was unchanged. Thus clinical observation alone based on therapeutic trial failed to indicate his digitalis status, with a view to further treatment. Consequently an acetyl strophanthidin test was carried out. A total of $0.85 \mathrm{mg}$. acetyl strophanthidin was administered over a period of 70 minutes. The results are shown in Fig. 1. Acetyl strophanthidin caused an abrupt slowing of the heart rate from 123 to 68 a minute. Extrasystoles were completely abolished. The patient experienced no nausea whatever during the test.

Interpretation. Digitalis administration was urgently required for the following reasons: (i) The sudden slowing of the heart rate suggests that the persistent tachycardia was due to an ectopic auricular pacemaker which was suppressed by acetyl strophanthidin, restoring sinus rhythm. Thus the tachycardia was not a feature of congestive failure per se., but rather an important aggravating cause of failure. (ii) The extrasystoles, although often bidirectional (Fig. 1) were not due to digitalis toxicity. They were abolished by acetyl strophanthidin when the heart rate was slowed. (iii) Nausea and vomiting were not due to the central emetic action of digitalis, but were the result of local gastro-intestinal changes associated with congestive failure, no doubt aggravated by local irritation of oral digitalis preparations. Consequently the patient was given digoxin intravenously to produce rapid digitalization and avoid nausea and vomiting. After 24 hours when $2.5 \mathrm{mg}$. digoxin had been administered, the pulse rate was slowed to 68 beats a minute, intraventricular conduction was improved, and no extrasystoles were noted. The patient's general condition improved. It was found possible to maintain digitalization by oral digoxin without nausea. Three weeks later after orthodox treatment for congestive failure he was able to get out of bed in comfort, and had not felt so well for months.

Case 2. Digitalis contra-indicated. A 59-year-old man was admitted with advanced congestive heart failure due to chronic bronchitis and emphysema. On examination he had anasarca, gallop rhythm, B.P. 160/90, serum potassium 4.7 m.eq. per litre. He had had repeated courses of digitalis and diuretic therapy for 18 months. Six weeks before admission he had been receiving $0.75 \mathrm{mg}$. digoxin orally each day and $2 \mathrm{ml}$. mersalyl twice weekly. But in spite of this treatment his condition deteriorated necessitating admission to hospital. He had no nausea or vomiting, and in spite of apparent adequate digitalis dosage there was sustained tachycardia. As his digitalis status was in doubt, an acetyl strophanthidin tolerance test was carried out; $1.8 \mathrm{mg}$. was administered over a period of one hour. Nausea and vomiting occurred, and the drug was immediately stopped. The heart rate was not significantly slowed, and very frequent multiform ventricular extrasystoles occurred, occasionally bidirectional.

Interpretation. Acetyl strophanthidin produced evidence of digitalis intoxication. Consequently further digitalis administration was omitted. All diuretics were stopped, and the patient given $5 \mathrm{~g}$. potassium chloride orally in divided doses each day for three days. The patient's general condition improved. Breathing was easier and a slower normal sinus rhythm supervened. Subsequently the treatment was resumed by oral digoxin $0.25 \mathrm{mg}$. twice daily and diuretics were again started. The patient continued to improve and cardiac compensation was better than it had been for many months.

Case 3. Combined digitalis and potassium therapy indicated. A man, aged 77 years, was admitted with advanced congestive heart failure due to coronary artery disease. He had rapid uncontrolled auricular fibrillation, B.P. 230/130, gallop rhythm and cardiac asthma. Serum potassium 4.5 m.eq. per litre. Various courses of digitalis and diuretic therapy of indeterminate quantity for two 


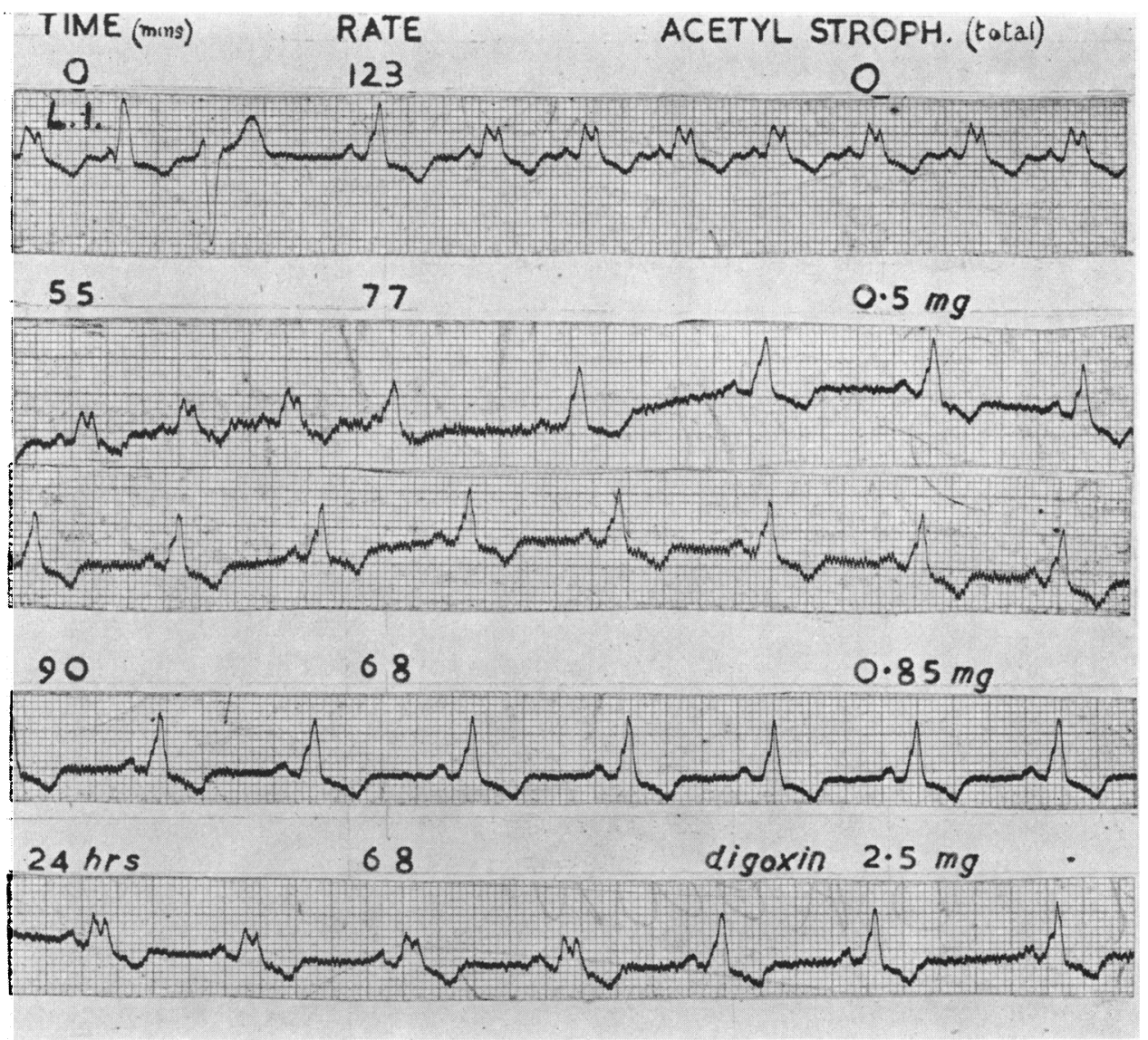

Fig. 1.-Case 1. Acetyl strophanthidin tolerance test. Therapeutic result. Restoration of sinus rhythm and abolition of ectopic beats.

years. He had been receiving $0 \cdot 25 \mathrm{mg}$. digoxin twice daily by mouth and choledyl, but, his doctor had to omit digitalis a few days before admission on account of nausea and vomiting. The patient felt that he was unable to tolerate any form of digitalis. Nevertheless on account of the uncontrolled auricular fibrillation digitalis foliata gr. i was resumed thrice daily along with $2 \mathrm{ml}$. mersalyl twice weekly. A week later there was no improvement. Tachycardia persisted, and nausea and vomiting reappeared. Consequently it was decided that an acetyl strophanthidin test should be carried out to determine the digitalis status of the patient with a view to rational therapy. When a small dose of $0.4 \mathrm{mg}$. acetyl strophanthidin had been administered the ventricular rate had slowed from 166 to $80 / 90$ beats a minute within half an hour (Fig. 2). The slowing was accompanied by the occurrence of very frequent multiform ventricular extrasystoles occasionally in groups. Acetyl strophanthidin administration was immediately stopped when the ectopic beats were noted, as they were the type considered typical of digitalis intoxication. No nausea was experienced during the test.

Interpretation. (i) The nausea and vomiting were not due to digitalis intoxication, but due to congestion of the abdominal viscera. (ii) Digitalis would slow the heart rate and would be required for that purpose. (iii) Digitalis might have produced extrasystoles and ventricular tachycardia. Consequently it was decided that digitalization should be carried out to slow the heart, and that potassium should be administered concurrently to suppress ectopic rhythms. Digitalis foliata gr. iii and potassium chloride $5 \mathrm{~g}$. were given orally daily in divided doses while diuretic therapy was suspended. Seven days later the pulse rate was 70 


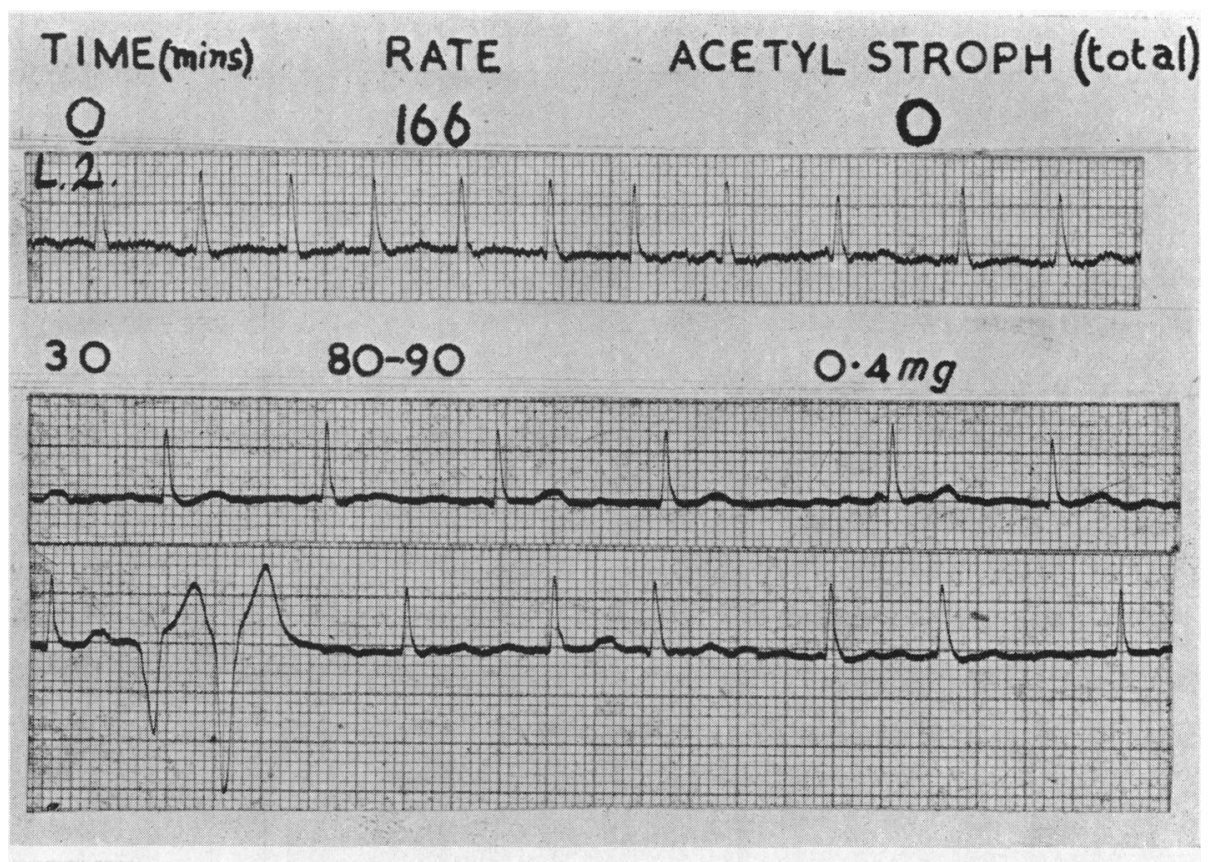

FIG. 2. Case 3. Acetyl strophanthidin tolerance test. Combined therapeutic and toxic result. Heart rate slowed but ectopic beats occur.

and no extrasystoles were noted under continual observation. Without further diuretic drugs, the congestive failure improved, and the patient was able to take digitalis without fear of nausea. Further improvement ensued with re-introduction of mercurial diuresis.

Case 4. Digitalis contra-indicated. A man, aged 50 years, was admitted with advanced congestive heart failure due to coronary artery disease. Gross anasarca and gallop rhythm, and frequent attacks of cardiac asthma. B.P. 130/80. Serum potassium 4.6 m.eq. per litre. Various courses of digitalis and diuretic therapy over a period of two years. Some weeks before admission he had been taking digitalis foliata gr. $\mathrm{i}$ thrice daily, but his condition deteriorated. As he had persistent tachycardia in spite of digitalis treatment an acetyl strophanthidin test was carried out as a guide to further management. When $0.7 \mathrm{mg}$. acetyl strophanthidin had been injected auricular and nodal extrasystoles appeared. After one hour when a total dose of $1.7 \mathrm{mg}$. had been given, multiform ventricular extrasystoles frequently occurred, and the test was then stopped (Fig. 3). The heart rate was not significantly altered.

Interpretation. Further digitalis administration would be likely to produce toxic arrhythmias which might be fatal. Moreover, no slowing of the pulse rate was likely before these toxic effects were manifest. Consequently digitalis and diuretic administration were suspended, and the patient was given potassium chloride $5 \mathrm{~g}$. orally each day in divided doses. Ten days later the patient's condition improved, and slow oral digitalization was instituted using digitalis foliata, and diuretic therapy was recommenced. The patient responded with slowing of the pulse rate and profuse diuresis and considerable clinical improvement.

Case 5. Digitalis indicated. A woman, aged 62 years, was admitted to hospital with advanced congestive heart failure due to coronary artery disease. Anasarca, gallop rhythm, and cardiac asthma. B.P. 150/80. Serum potassium $4.5 \mathrm{~m}$.eq. per litre. She had a history of recurrent heart failure during the previous five years with varying courses of digitalis and diuretic therapy. Previous hospital admissions confirmed that at times she had pulsus bigeminus requiring interruption of digitalis treatment. One week before admission, digitalis had to be omitted on account of intense nausea and vomiting, and the occurrence of coupled beats. As she had rapid auricular fibrillation 


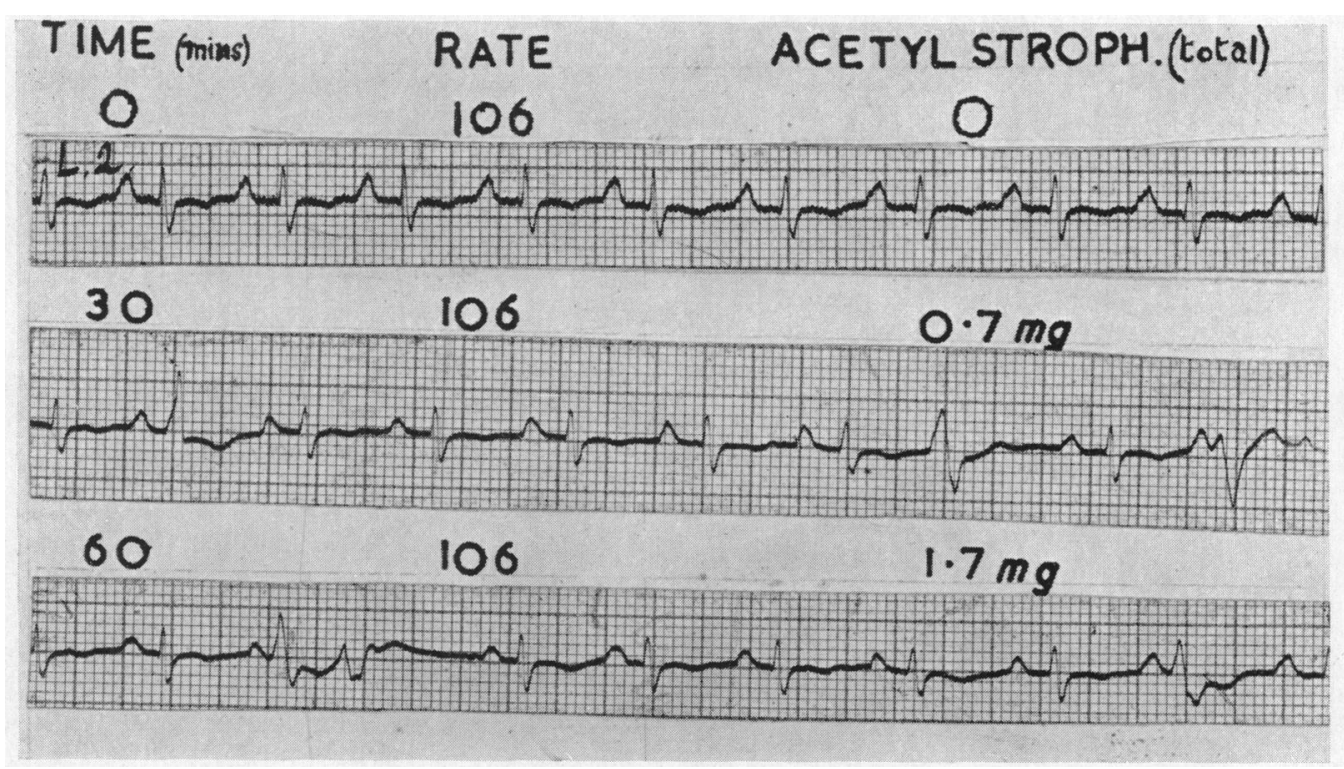

FIG. 3.-Case 4. Acetyl strophanthidin tolerance test. Toxic result. Occurrence of ectopic beats.

(rate 150 a minute) and in view of the previous history, an acetyl strophanthidin tolerance test was carried out to help determine her digitalis status. When $0.5 \mathrm{mg}$. acetyl strophanthidin had been administered the ventricular rate showed evidence of slowing, and when a total of $1 \mathrm{mg}$. had been given over a period of one hour, the auricular fibrillation was controlled (rate 60-75 a minute). No nausea or any other untoward effect was observed.

Interpretation. The slowing of the heart rate by acetyl strophanthidin appeared to indicate that complete rapid digitalization was required. As the patient was apprehensive about taking digitalis by mouth on account of nausea, digoxin was given by the intravenous route. Within 48 hours when a total of $2.5 \mathrm{mg}$. had been administered, the heart rate was 60-70 a minute. This was accompanied by considerable improvement, and it was found possible to maintain digitalization with oral digoxin without undue nausea. When diuretic therapy was recommenced, there was a satisfactory response. Four weeks later the patient was well enough to be out of bed.

Case 6. Digitalis indicated. A man, aged 59 years, was admitted with advanced congestive heart failure due to coronary artery disease (cardiac infarction four years previously). During the last two years he had recurrent episodes of congestive failure with varying courses of digitalis and diuretic therapy. He had been confined to bed for two months and was receiving $0 \cdot 25 \mathrm{mg}$. digoxin twice daily and $2 \mathrm{ml}$. mersalyl twice weekly. But he failed to improve, and admission to hospital became necessary. There was rapid auricular fibrillation with frequent ventricular extrasystoles. In view of the ectopic beats, the possibility of digitalis toxicity was entertained. Consequently an acetyl strophanthidin tolerance test was carried out to determine his digitalis requirements. The results are shown in Fig. 4. Two hours later a total of $2.2 \mathrm{mg}$. of the drug had been administered, the heart rate was slowed from 95-100 to 60-75 a minute and the ventricular extrasystoles were completely abolished.

Interpretation. Digitalis was required for this patient to slow the heart rate. The extrasystoles were a manifestation of myocardial disease and not due to digitalis. Consequently oral digitalization was instituted by digoxin $0.25 \mathrm{mg}$. six-hourly with diuretic therapy. One week later the pulse rate was 80 a minute and there was considerable clinical improvement.

Case 7. Digitalis indicated. A man, aged 66 years, was admitted with advanced congestive heart failure due to chronic rheumatic heart disease. He had rapid auricular flutter. Before admission he had been receiving digoxin $0.25 \mathrm{mg}$. thrice daily by mouth and diuretic therapy for many months. 


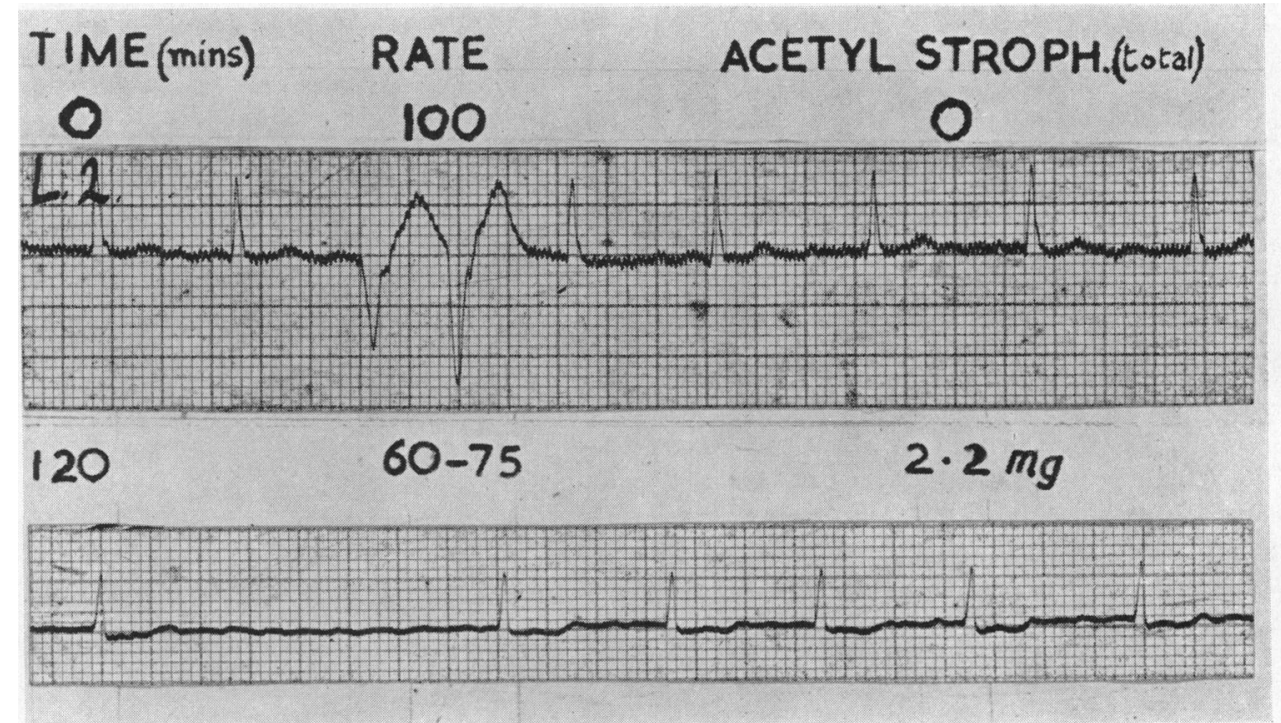

FIG. 4.-Case 6. Acetyl strophanthidin tolerance test. Therapeutic result. Heart rate slowed and ectopic beats abolished.

B.P. 150/80. Serum potassium 6.4 m.eq. per litre. He complained of nausea but nevertheless was able to tolerate oral digitalis. In view of the rapid ventricular rate, and the failure of the digitalis dosage to effect control, an acetyl strophanthidin tolerance test was carried out to determine the possible response to further digitalis administration. Fig. 5 shows the result. Four hours

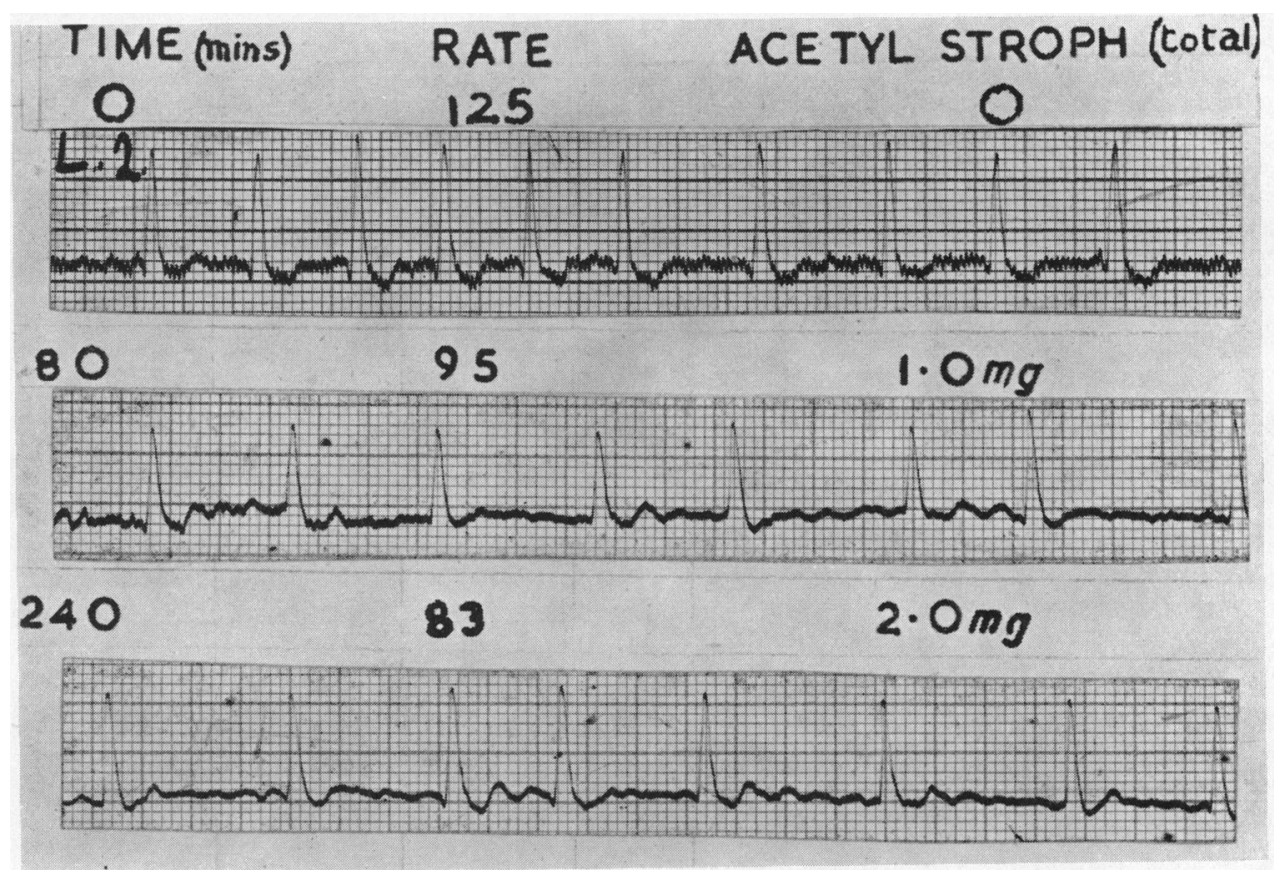

FIG. 5.-Case 7. Acetyl strophanthidin tolerance test. Therapeutic result. Heart rate slowed. 
after $2 \mathrm{mg}$. of the drug had been given the heart rate had fallen from 105-140 to 80-100. No nausea or vomiting occurred during the test.

Interpretation. Digitalis was indicated to control the heart rate. The patient's nausea was due to congestive changes and not to digitalis intoxication. Consequently digitalis foliata was administered in doses of gr. i four-hourly until adequate slowing of the heart was obtained.

Case 8. Digitalis indicated. A man, aged 63 years, was admitted with congestive heart failure due to coronary artery disease. He had anasarca and pulmonary œdema. B.P. 190/115. Serum potassium 4.6 m.eq. per litre. He had received digitalis and diuretic therapy at varying times during the previous six years. For two months he had been confined to bed at home, and was receiving digitalis foliata gr. $i$ thrice a day along with diamox therapy. However, it had to be omitted as he had nausea and vomiting and extrasystoles. In view of these factors it was decided to carry out an acetyl strophanthidin test. At the end of three hours when a total of $1.8 \mathrm{mg}$. acetyl strophanthidin had been administered, the heart rate was slowed from 130-150 to 80-90 a minute. The extrasystoles were abolished with the slowing of the heart rate. No nausea or vomiting occurred during the test.

Interpretation. Digitalis was required to slow the heart rate. The nausea and vomiting were due to local congestive changes and not to digitalis. The extrasystoles were due to the underlying heart disease and not to digitalis toxicity. Consequently digitalization was carried out using intravenous digoxin, to avoid nausea by the oral route. After $1.5 \mathrm{mg}$. digoxin had been given the pulse rate fell to 84 a minute. No extrasystoles occurred. Digitalization was maintained by oral digoxin without undue nausea.

Case 9. Combined digitalis and potassium therapy indicated. A woman, aged 63 years, was admitted to hospital with congestive heart failure due to coronary artery disease (an old posterior infarction). She had auricular fibrillation and pulmonary œdema with hydrothorax. There was a history of congestive failure of varying degree over a period of two years. No information was available about the amount of digitalis she had had prior to admission. On account of the uncontrolled auricular fibrillation $0.25 \mathrm{mg}$. digoxin was administered daily by mouth. After a few days' treatment, she developed intense nausea and vomiting. The digitalis was omitted for a few days, but when recommenced the nausea promptly recurred. Her pulse rate continued to be rapid. It was decided thereafter to carry out an acetyl strophanthidin tolerance test as a guide to her digitalis requirements. The lead selected (AVF) confirmed the presence of auricular fibrillation and posterior infarction (Fig. 6.) When only $0.4 \mathrm{mg}$. acetyl strophanthidin had been given, the heart rate significantly slowed from 125 to 95 , though this was accompanied by frequent ventricular extrasystoles. No nausea occurred during the test.

Interpretation. Digitalis was indicated to slow the heart rate. However, as the slower rate was likely to be associated with extrasystoles, potassium was indicated to suppress them. Consequently the patient was digitalized with digitalis foliata gr. i. thrice daily and potassium chloride $5 \mathrm{~g}$. daily was given orally. A week later the pulse rate was 70 , and no extrasystoles were noted. The patient's condition was considerably improved by adequate digitalis treatment.

\section{DisCUSSION}

All the cases were in the older age group and coronary artery disease was the prevalent underlying cause of intractable failure. It was probably also a factor in Cases 2 and 7 . With an ageing population such cases are likely to increase, and any help with their management is opportune. The wide range in dosage of acetyl strophanthidin $(0.4-2.2 \mathrm{mg}$.) required to produce a result indicates the difficulty of assessing adequate digitalis dosage based on clinical observation only. Moreover, it is reasonable to assume that when a therapeutic effect occurs, the amount of acetyl strophanthidin administered may be a guide to the dosage of conventional digitalis preparations required for control of the pulse rate. The clinical features of Cases 2 and 4 in which digitalis was contraindicated differed little from the remaining cases in which digitalis was urgently required. This difficulty is a characteristic feature of intractable heart failure. Perusal of the case histories in our series reveals the inadequacy of digitalis treatment prior to the acetyl strophanthidin tolerance 


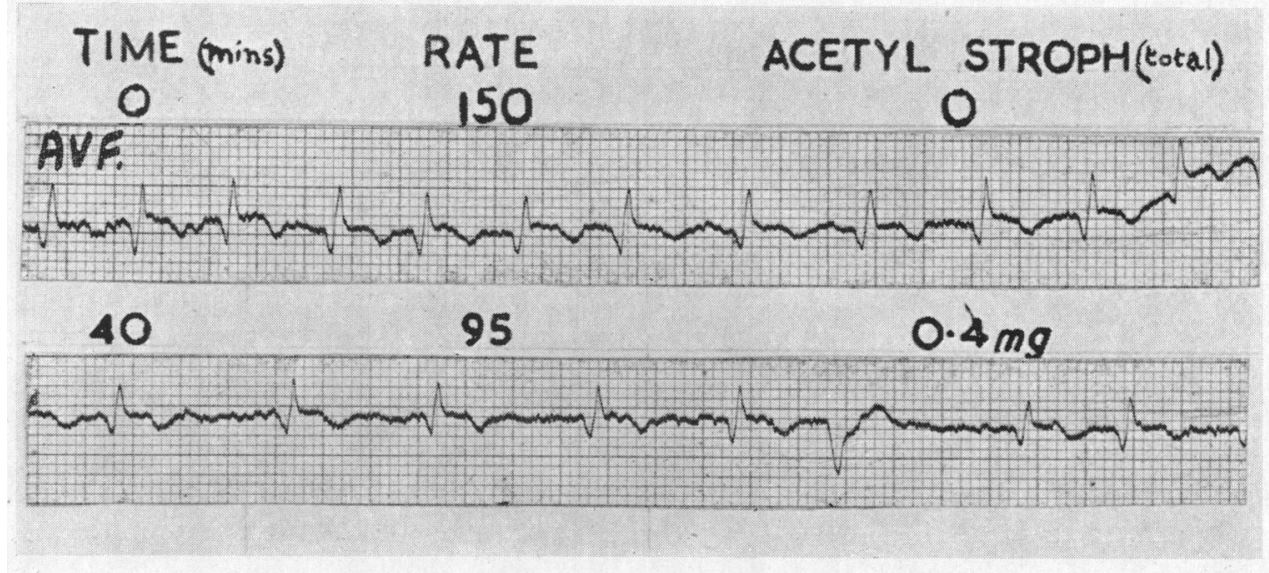

FIG. 6.-Case 9. Acetyl strophanthidin tolerance test. Combined therapeutic and toxic result. Heart rate slowed but ectopic beats occur.

test. Nausea may preclude further oral therapy. Some of our patients refused to take digitalis by mouth. Tachycardia and ectopic rhythms are responsible for varying opinions about what should be done and as a result "therapeutic half measures" are usually adopted. Digitalis may be given, then omitted, then recommenced with varying dosage. Such measures are not only undesirable but may be dangerous. The acetyl strophanthidin tolerance test represents a step away from empiricism in digitalis management in these cases, as it affords objective evidence on which treatment can be based with greater confidence.

We wish to draw attention to the qualitative difference between the therapeutic and toxic action of digitalis on the diseased heart. Whereas therapeutic action slows the heart by vagal stimulation and increasing the cardiac output, toxic action produces ectopic beats due to local effect of the drug on isolated groups of cardiac cells. This is in keeping with modern theory concerning extrasystolic impulse formation (Scherf and Schott, 1953). Our results have been interpreted on this basis. It is notoriously difficult to produce pulsus bigeminus by the pharmacological action of digitalis on the normal heart, in contra-distinction to its very frequent occurrence when the heart is diseased (Scherf and Schott, 1953). The results of our investigations appear to indicate that in the presence of heart disease there is competition between the therapeutic action of digitalis on the heart as a whole, and its toxic action on isolated groups of cardiac cells causing ectopic rhythms. In Cases 2 and 4 acetyl strophanthidin produced multiform ventricular extrasystoles without alteration in pulse rate, i.e. the toxic action was predominant. In the seven other cases it slowed the heart rate though in some instances this was accompanied by a toxic action causing ectopic beats (Cases 3 and 9). In Cases 1, 6 and 9 acetyl strophanthidin not only slowed the heart rate, but thereby abolished ventricular extrasystoles. The explanation of this paradoxical action of the drug on ectopic foci appears to be that the longer diastolic pause at slower heart rates permits more efficient coronary filling, thereby relieving myocardial anoxia which is responsible for ectopic beats when the heart rate is fast. Thus digitalis can produce a therapeutic effect, a toxic effect, or a combination of both, and this interplay between the therapeutic and toxic action explains the varying response of the diseased heart to digitalization. Our experience shows that the smaller the amount of digitalis that produces toxic arrhythmias, the more severe is the underlying cardiac disease. When digitalis produces only toxic manifestations, the final stage of myocardial breakdown has been reached.

Acetyl strophanthidin is a potent drug, and ventricular fibrillation has been reported during its use (Enselberg et al., 1951). Considerable experience is required for the selection of cases suitable for a test dose. Fresh myocardial infarction should be excluded. Serial electrocardiographic 
control is essential, and the observer must be competent to recognize immediately the significance of the changes in rhythm. In our series, the objective was to administer the minimal amount of the drug to produce a response. A full therapeutic or toxic effect was not necessarily produced. All our cases had tachycardia (4 auricular fibrillation, 3 sinus tachycardia, 1 auricular flutter, 1 auricular tachycardia). However, the basic rhythm must be analysed electrocardiographically to exclude toxic digitalis tachycardia such as ventricular tachycardia, and auricular tachycardia with A-V block (Heyl, 1932). Disturbance of A-V conduction is also a contra-indication to the test. Nausea and vomiting is not a contra-indication. It is important to note that six of our patients (Cases 1, 3, $5,7,8,9)$ who had nausea as a prominent symptom did not manifest an emetic response to acetyl strophanthidin. Only one (Case 2) developed nausea and vomiting during the test. In intractable failure the nausea associated with visceral congestion seriously interferes with adequate digitalization maintenance. It cannot be differentiated from the central emetic action of digitalis except by intravenous therapeutic trial such as with acetyl strophanthidin. No subjective paræsthesiae occurred during the test in any of our patients, as noted by Lown and Levine (1955). With the technique used no necessity for procaine amide administration arose. Moreover, the risk of the test is less than the risk of administering digitalis in the presence of potential digitalis poisoning, or failing to administer the drug when it is urgently required.

Apart from intractable heart failure, the acetyl strophanthidin tolerance test may be of great value in assessing digitalis requirements when the sensitivity of the heart to digitalis has been altered by potassium loss associated with surgical operations. A digitalized patient may require adjustment of dosage following operation due to vomiting or dietetic restriction. Lown and Levine (1955) describe the use of the test after valvotomy. Thus it opens a new field in the post-operative management of cardiac patients receiving digitalis.

Certain important deductions can be made concerning digitalis treatment schedules based on the results of the acetyl strophanthidin tolerance test. Diuretic therapy is suspended until the digitalis requirement has been ascertained. If a toxic response alone occurs (Cases 2 and 4) then, of course, digitalis is omitted, and potassium administered to suppress the activity of ectopic foci. We have used oral potassium in dosage of $5 \mathrm{~g}$. daily. Usually after two to five days the potassium balance is adequately restored to allow recommencement of digitalis therapy, and dosage which was previously toxic came within the therapeutic range. None of our patients suffered undue nausea from oral potassium treatment in spite of intense nausea when digitalis was given. When a therapeutic response only is obtained, then obviously, digitalis must be given (Cases $1,5,6,7,8)$. Important principles then arise concerning dosage and route of administration. No digitalis treatment is attempted until at least ten hours after the trial dose of acetyl strophanthidin to avoid a combined action of the drugs. Adequate oral digitalis dosage by mouth may be impossible on account of nausea and vomiting. Urgent action may be imperative and in such cases we have digitalized our patients using the intravenous route (Cases $1,5,8$ ) basing the dose of digoxin on the amount of acetyl strophanthidin used for the test. We believe that this measure was life-saving in our cases, and the view that digitalis should never be given intravenously when the patient has been already taking the drug by mouth may require revision. Our view is that intravenous digitalis therapy may be urgently indicated in such cases and may be the only effective route to produce maximum benefit in a limited period of time. In Cases 3 and 9 a combined therapeutic and toxic effect occurred when acetyl strophanthidin was given. We regard this result as an indication for combined treatment with digitalis and potassium, to enable the patient to have the benefit of the therapeutic action of the drug without toxic manifestations. Potassium chloride $5 \mathrm{~g}$. is given orally daily, concurrently with digitalis, usually during the first two to five days. Serial electrocardiographic study is essential when potassium is given to adjust dosage and prevent toxic effects. It should be noted that serum potassium levels are no guide to treatment. In most of our cases they were within the normal range. It is a negative potassium balance that determines increased sensitivity to digitalis, and obviously patients with intractable heart failure requiring urgent treatment are not suitable for complete metabolic study. Combined digitalis and potassium treatment in selected cases is a therapeutic 
advance. The use of digitalis following fresh infarction may be associated with serious toxic arrhythmias. If it is combined with potassium administration, toxic manifestations may be suppressed, so permitting the patient to benefit from its therapeutic action. This problem is now under investigation by us.

\section{SUMMARY}

The complex factors in relation to digitalis therapy in intractable heart failure are discussed, especially in relationship to potassium metabolism. The acetyl strophanthidin tolerance test is described and applied to nine patients. The results obtained indicate new principles of digitalis treatment based on the concept of competition between its therapeutic and toxic actions on the diseased heart. Parenteral administration of digitalis is entirely justifiable if indicated by the test dose of acetyl strophanthidin, irrespective of previous dosage of the drug, especially if nausea prevents oral treatment. Combined digitalis and potassium therapy represents a therapeutic advance in selected cases. Tachycardia may occur with serious digitalis toxicity, and in many instances the acetyl strophanthidin test alone can differentiate it from tachycardia requiring urgent digitalis treatment.

\section{REFERENCES}

Berliner, R. W., Kennedy, T. J., and Orloff, J. (1951). Amer. J. Med., 11, 274.

Burrows, B. A., and Sisson, J. H. (1950). J. Clin. Invest., 29, 801.

Calhoun, J. A., and Harrison, T. R. (1931). J. Clin. Invest., 10, 139.

Chen, K. K., and Elderfield, R. C. (1942). J. Pharmacol. exper. Therap., 76, 81.

Dennis, J., and Moore, R. M. (1938). Amer. J. Physiol., 123, 443.

Eggleston, C. (1915). Arch. intern. Med., 16, 1.

Enselberg, C. D., Croce, J. P., and Lown, B. (1951). Circulation, 3, 647.

Gold, H., Otto, H. L., Modell, W., and Halpern, S. L. (1943). J. Pharmacol. exper. Therap., 86, 39.

Greiner, T., and Reilly, J. (1952). Proc. Soc. exper. Biol., 81, 141.

Harvey, R. H., Ferrer, M. I., Cathcart, R. T., Richards, D. W., and Cournand, A. (1949). Amer. J. Med., 7, 439.

Hatcher, R. A., and Weiss, S. (1922). Arch. intern. Med., 29, 690.

Heyl, A. F. (1932). Ann. intern. Med., 5, 858.

Lesser, G. T., Dunning, M. F., Epstein, F. H., and Berger, E. Y. (1952). Circulation, $5,85$.

Levine, H. D. (1948). Ann. intern. Med., 29, 822.

Lown, B., and Levine, S. A. (1955). Current Concepts in Digitalis Therapy. Churchill.

, Salzberg, H., Enselberg, C. D., and Weston, R. E. (1951). Proc. Soc. exper. Biol., $76,797$.

Root, M. A., and Chen, K. K. (1953). Proc. Soc. exper. Biol. 84, 131.

Sampson, J. J., Alberton, E. C., and Kondo, B. (1943). Amer. Heart J., 26, 164.

Scherf, D., and Schott, A. (1953). Extrasystoles and Allied Arrhythmias. Heinemann. 